\title{
A STABLE AND SIMPLE METHOD OF "FAGLUPAGAS FIXATION" FOR GATEGHOLAMINES FOR ROUTINE EXAMINATION
}

\author{
Harumichi IMAI, Hiroshi KIMURA and Toshiniro MAEDA \\ Department of Anatomy, Shiga University of Medical Science, Otsu 520-21
}

Received for publication July 5, 1982 and in revised form August 3, 1982

\begin{abstract}
Various techniques of fluorescence histochemistry have some methodological difficulties mainly due to the instability of amine fluorophores. The present study aims to develop a stable and relatively simple histofluorescence method which can be applied to the routine examination for diagnostic histopathologists.

Dissected tissue pieces were fixed by immersing in a FAGLUPAGAS* solution overnight. Cryostat sections of fixed materials were washed in a FAGLUGAS solution, mounted on glass slides, dried by warm air and finally cover-slipped in Entellan.

Amine fluorescences in thusly fixed tissues were stable for several months when the specimens, either tissue blocks or cryostat sections, were kept in the FAGLUPAGAS.
\end{abstract}

The methods of fluorescence histochemistry for bioactive monoamines are roughly divided into two groups, a dry type and a wet type. The most common and original dry type technique is represented by the freeze-drying-FA vapor method developed by Falck-Hillarp (3). The wet type method, which does not need to process materials with a freeze-drying procedure, was first introduced by using glyoxylic acid (7) as a reactive acid aldehyde in Pictet-Spengler condensation. Later, it was also developed by Furness et al. (4) in another line based on the condensation reaction in a mixture of formaldehyde and glutaraldehyde to produce a water stable amine fluorophore. Although the dry type technique can be achieved by a simple procedure and allows reproducible visualization of fluorogenic amines, several improvements for increasing sensitivity have been reported by such fixatives as glyoxylic acid or aluminum salt. Likewise, the wet-type method includes several modifications by, for example, the use of metalic ion linkage (see ref. 1). The demonstration of amine fluorescence has yet several technical difficulties, especially in the preparation of sections during which critical attention is required for temperature, humidity, and the duration of fixation. Among them the most critical factor is the duration of section processing, so that previous techniques are extremely difficult for the application to materials obtained in clinical cases such as biopsy,

* FA: paraformaldehyde (4\%), GLU: glutaraldehyde $(0.5 \%)$, PA: picric acid $(0.2 \%)$, GA: glyoxylic acid $(2 \%)$, S: sucrose $(15 \%)$. 
autopsy or surgical operation.

In this paper a new modification of the wet-type amine fluorescence technique is described which yields stable fluorophores and is flexible in periods of the procedure. Results obtained by this method are given in the peripheral nervous system of several human organs and in the central nervous system of the rat.

\section{MATERIALS AND METHODS}

Several organs of human materials excised by surgical operation were obtained from the Wakayama Medical School. Male Wistar rats weighing 150-200 g were used. Various conditions of fixation were examined with different fixative mixtures containing paraformaldehyde (FA), glutaraldehyde (GLU), picric acid (PA), and glyoxylic acid (GA) in different concentrations.

In our preliminary study, it was found that tissue blocks can be fixed sufficiently for preserving amines by immersion fixation when they are cut into slabs less than $1 \mathrm{~cm}$ thick.

Procedure

1) Immerse tissue blocks (less than $1 \mathrm{~cm}$ thick) in the following FAGLUPAGAS solution overnight (or for up to several months), $2-4^{\circ} \mathrm{C}$.

\section{FAGLUPAGAS SOLUTION}

i) Dissolve $2 \mathrm{~g}$ glyoxylic acid (GA) in $50 \mathrm{ml}$ of phosphate buffer, $0.2 \mathrm{M}$ (PBa), $\mathrm{pH} 7.4)$.

ii) Adjust $\mathrm{pH}$ to 7.4 with $5 \mathrm{~N}-\mathrm{NaOH}$.

iii) Add $20 \mathrm{ml}$ paraformaldehyde ( $\mathrm{FA}^{\mathrm{b})}, 20 \%$ in water),

$2 \mathrm{ml}$ glutaraldehyde (GLU, 25\% commercially obtained),

$15 \mathrm{ml}$ picric acid (PA, $1.33 \%$ saturated trinitrophenol $\left.{ }^{\mathrm{c}}\right)$, and $15 \mathrm{~g}$ sucrose

2) Section with a cryostat $(10-20 \mu \mathrm{m})$.

3) Immerse the sections in FAGLUPAGAS solution for 20 min (or for up to one month), $2-4^{\circ} \mathrm{C}$.

4) Immerse these sections in FAGLUGAS solution for 20 min (or for up to $24 \mathrm{hr}$ ), $2-4^{\circ} \mathrm{C}$.

5) Mount on chrome-coated glass slides.

6) Dry with a hair dryer $\left(40-60^{\circ} \mathrm{G}\right)$ for $20 \mathrm{~min}$.

7) Embed in Entellan without dehydration with ethanol (xylene may be used if necessary).

Tissue sections thus prepared were observed under an Olympus incident fluorescent microscope equipped with a dichronic-mirror of $\mathrm{V}$ system providing an excitation wave length of $405 \mathrm{~nm}$.

\footnotetext{
a) $143.3 \mathrm{~g} \mathrm{Na}_{2} \mathrm{HPO}_{4}$ and $15.6 \mathrm{~g} \mathrm{NaH}_{2} \mathrm{PO}_{4}$ in $2,500 \mathrm{ml} \mathrm{H}_{2} \mathrm{O}$.

b) $20 \mathrm{~g}$ paraformaldehyde powder in $100 \mathrm{ml} \mathrm{H} \mathrm{H}_{2} \mathrm{O}$. Heat at $70^{\circ} \mathrm{C}$ for $3 \mathrm{hr}$, add $0.1 \mathrm{ml}$ of $1 \mathrm{~N}$ $\mathrm{NaOH}$, the solution will become clear after cooling down. Filtrate with No. 2 filter paper.

c) Do not try to use excess amount of PA for saturation, measure exactly the weight of PA and heat at $40^{\circ} \mathrm{C}$ (not to exceed $45^{\circ} \mathrm{C}$ ).

d) FAGLUGAS solution

Omit PA from the above "FAGLUPAGAS" solution.

e) Should completely wash out yellow color of PA.
} 
The emission spectra of amine fluorescence were examined in a Carl Zeiss SF microspectrophotometer equipped with stepwise scanning system of serial interference filters from $400 \mathrm{~nm}$ to $610 \mathrm{~nm}$ and with HP 975 calculator. The emission spectral curve was calibrated by a YHP 45 computer.

\section{RESULTS}

\section{Characteristics of fluorophore by FAGLUPAGAS}

Figure 1 shows the emission spectra of fluorescent compounds produced by the FAGLUPAGAS fixative in the cell bodies of the locus coeruleus of the rat brain. The emission peak at $470-480 \mathrm{~nm}$ was identical with that obtained by other fluorescence histochemical techniques employing different condensation the procedures such as with the formaldehyde vapour, GA solution and FAGLU solution $(4,5,7)$. This green fluorescence of the aminergic cells was completely diminished after treatment with sodium borohydride solution (2), and was regenerated by immersing sections again in the GAS solution. These results indicated that the fluorescence with this technique was specific for biogenic catecholamines.

\section{Animal tissues which can be fixed by perfusion}

Although this FAGLUPAGAS solution is devised for an immersion fixative, a perfusion fixation prior to the immersion fixation is recommended to obtain more stable results when examining animal brains or human organs large enough for perfusion. For this purpose a FAGLUPAGA, subtracted sucrose from the FAGLUPAGAS, should be used for a perfusion fixative. Thus fixed tissues were then processed according to the above-mentioned procedure from the first step.

Noradrenaline (NA)-containing cell bodies in the locus coeruleus (Fig. 2a), and dopamine (DA)-containing cell bodies in the substantia nigra (Fig. 2b) of the rat brain showed intense amine fluorescences, respectively. These amine fluorescences were found in perikarya of these cells whose nuclei were devoid of fluorophores. No signs of diffusion of either amines from somata appeared to exist even after the specimens were fixed for long periods.

In addition, NA fibers were observed with a high sensitivity. The rat cerebel-

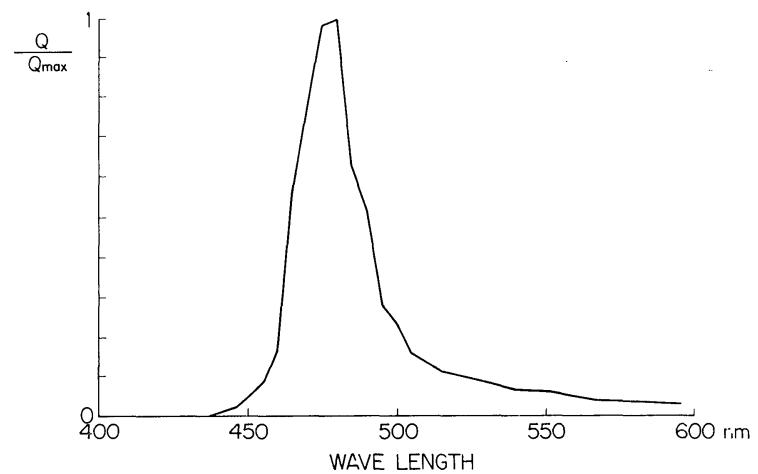

FIG. 1. Emission spectrum by microspectrofluorophotometry of the fluorophore with FAGLUPAGAS fixative in a cell body of the locus coeruleus of rat brain. Maximum wave length $=470-480 \mathrm{~nm}$. 

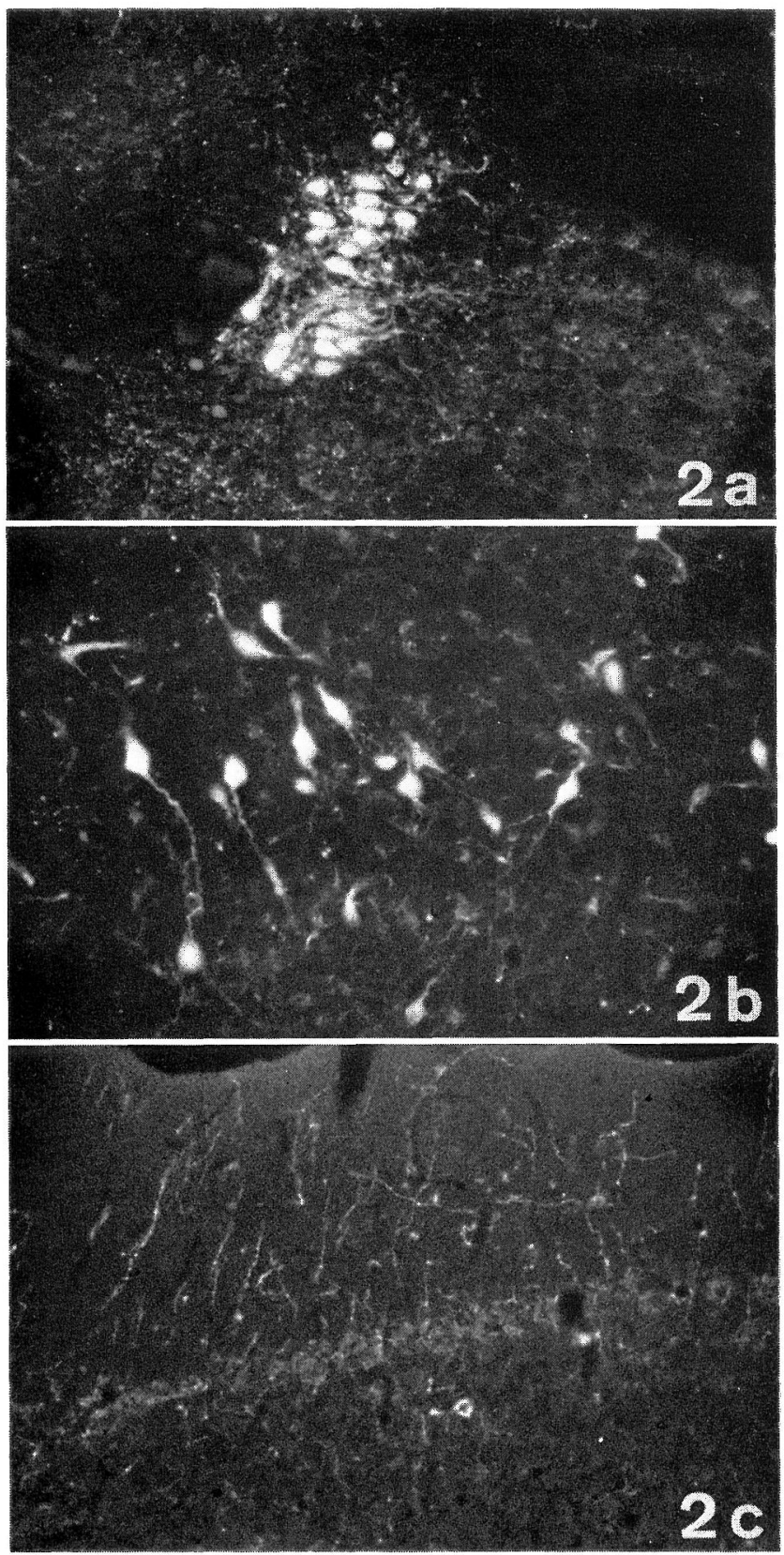

Figs. 2a-c. Rat brain. Very intense fluorescence of noradrenaline in somata of the locus coeruleus (Fig. 2a), of dopamine in somata of the substantia nigra (Fig. 2b), and of noradrenaline in nerve fibers of the cerebellar cortex (Fig. $2 \mathrm{c}$ ) $. \quad \times 110$ 
lum, for example, which has been known to contain a very low amount of NA, had many varicose amine fibers throughout this structure and an example is presented with the cerebellar cortex (Fig. 2c). In the red nucleus of the stria terminalis, fine amine fibers, probably of DA, were observed. Although very brilliant DA fluorescence was present in the neostriatum of the rat, no detailed structure of DA fibers could be visualized. The detection of these fine DA fibers, therefore, was not within the limits of this method.

Human materials which can be fixed only by immersion

Small tissue blocks ( $1 \mathrm{~cm}$ thick) were immersed in the fixative of FAGLUPAGAS within $30 \mathrm{~min}$ after excision, and were stored overnight. After sectioning with a cryostat, these sections were treated as described in the methods, and were examined under a fluorescence microscope.

In the stomach, varicose fibers of blue-green fluorescence were found in both the deep portion of the mucous membrane layer and the submucous layer, and some of them were observed to innervate the superficial layer adjacent to the epithelium (Fig. 3a). In addition, scattered cell groups, one group consisting of about 10 somata containing amine fluorescence, were seen in the submucous layer invading the deep layer of the mucous membrane (Fig. 3b). These somata were identified to be intramural ganglion cells. Moreover, small cells situated around the gastric glands emitted yellow fluorescence, which may be identical to that of serotonin contained in the enterochromaffin cells. Indeed, this type of fluorescence exhibited the highest intensity at the emission wave length adjusted to $510 \mathrm{~nm}$, and thus was very likely serotonin (Fig. 3c).

In a part of nearly intact mucous membrane of the gall bladder, fine noradrenaline fibers with varicosities were found within the propria mucosae and adjacent to invaginations of the lining epithelium (Rokitanski-Aschoff sinus) (Fig. 4a). The wall around the inflammatory area of this gall bladder had a very high density of varicose amine fibers making thick bundles (Fig. $4 \mathrm{~b}$ ), indicating an accumulation of amines within these fibers. They were loosely distributed in areas neighbouring necrotic tissues (Fig. 4c). This phenomenon suggests that peripheral aminergic neurons are affected by or are fundamentally related to the inflammation.

Comparison of amine fluorescences with various fixative mixtures.

A solution of FAGLUS gave a fairly stable result among several fluorescence histochemical techniques of the wet type. However, incubation longer than 24 $\mathrm{hr}$ in this fixative damaged amine specific fluorescence and, in turn, gave higher background (non-specific) fluorescences. These demerits were significantly improved by adding PA to this solution to make up a FAGLUPAS solution, and better results were obtained also with regard to the intensity and stability of amine fluorescences (Table 1). This was particularly true with amine-containing perikarya in

Figs. 3a-c. The body of stomach, an operated human material. Many fibers with green noradrenaline fluorescence run in the propria mucosae between gastric glands and in the submucosa (asterisk) (Fig. 3a). Fig. 3b shows intramural ganglion cells located in the deep region of the propria mucosae close to the base of the gastric glands. The muscularis mucosae is densely innervated by noradrenaline varicose fibers. Fig. $3 \mathrm{c}$ shows enterochromaffin cells with yellow serotonin fluorescence found in a middle portion of the gastric glands. Some fine noradrenaline fibers are also seen. (Barrier filter; $515 \mathrm{~nm}$ ). $\times 200$ 






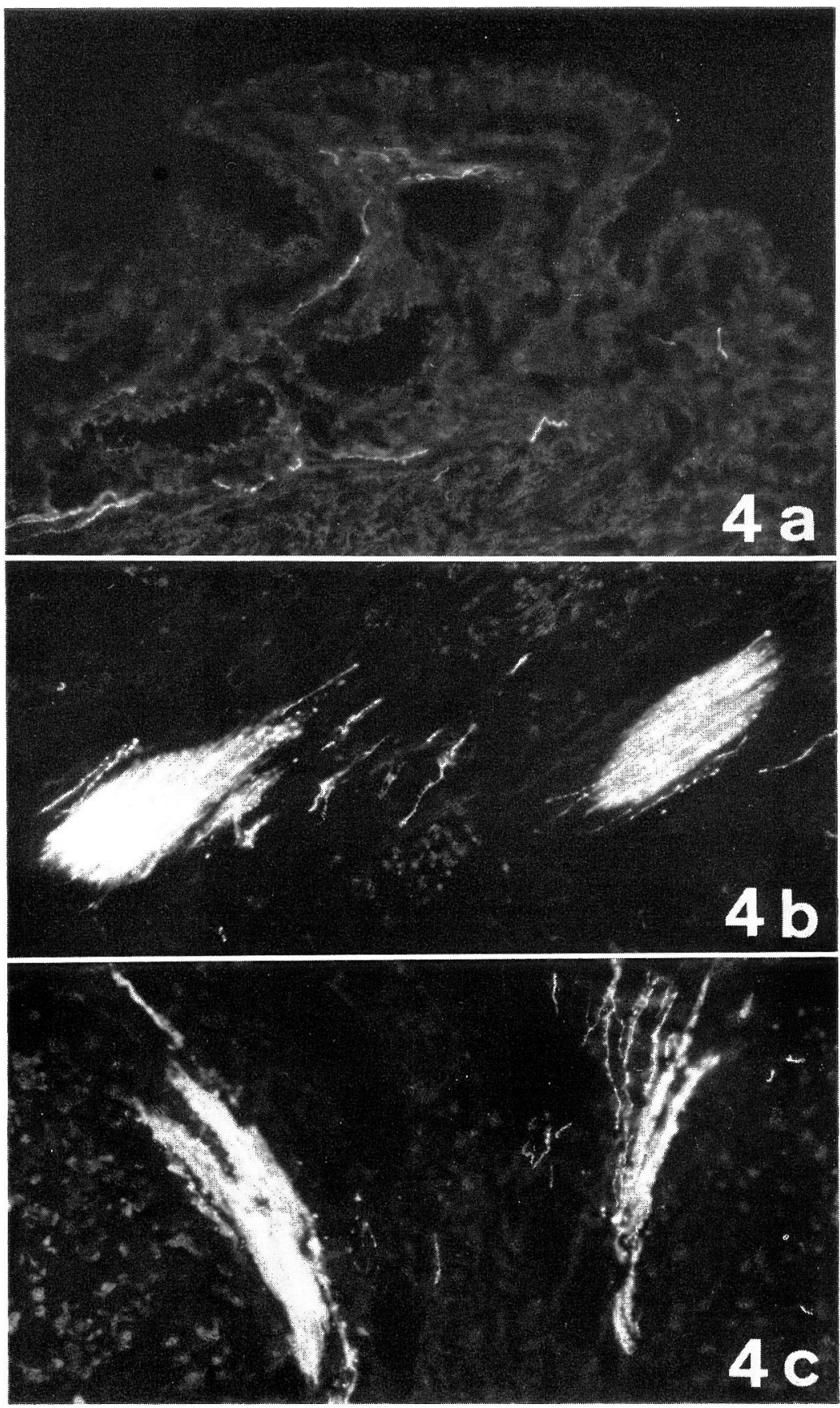

Frgs. 4a-c. Human gall bladder, operated material of cholecystitis. a; normal mucosa, b; inflammatory area, $\mathrm{c}$; necrotic tissue. $\times 200$ 
TABLE 1. Comparison of amine fluorescence with various fixative mixtures

\begin{tabular}{|c|c|c|c|c|c|c|c|c|}
\hline \multirow{3}{*}{ solution II } & \multicolumn{8}{|c|}{ solution I } \\
\hline & \multicolumn{2}{|c|}{ FAGLUS } & \multicolumn{2}{|c|}{ FAGLUPAS } & \multicolumn{2}{|c|}{ GAS } & \multicolumn{2}{|c|}{ FAGLUPAGAS } \\
\hline & soma & fiber & soma & fiber & soma & fiber & soma & fiber \\
\hline FAGLUS & H & + & $H$ & + & t & + & $H$ & + \\
\hline GAS & + & + & H & H & + & H & H & H \\
\hline FAGLUGAS & $H$ & + & HH & H & + & + & H & H \\
\hline
\end{tabular}

Solution I: A fixative for immersion fixation of the first step.

Solution II: A fixative for immersion fixation of the second step to remove PA yellow color from sections. +: weak, H: moderate, H: intense.

the rat brain. However, a certain extent of diffusion occured in fluorescent nerve fibers and terminals, thus the results obtained with the FAGLUPAS fixative was generally inferior to those with vibratome sections fixed by GA only. Although this GA method gave the known highest sensitivity, it was very difficult in such technical procedures as sectioning or drying specimens.

The FAGLUPAGAS solution, on the other hand, permitted visualization of various amine-containing structures in greater detail than the above fixatives, with intense fluorophores relatively resistant against photo-decomposition. Furthermore, these amine-related fluorophores were well preserved for a long period at least several months when specimens, either tissue blocks or sections, were kept in this fixative.

\section{DISCUSSION}

The present technique appeared to have almost the same value as the conventional freeze-drying method. It has shown satisfactory results for visualizing monoamine containing structures in various organs including brains of the human and the rat, with an exception of fine DA fibers in the rat striatum. Due to the simple procedure and the resulting stable fluorophores, this method is suitable for examinations of specimens from operation or biopsy.

Picric acid (PA), which played the most important role in the new fixative, has been used also in fixatives for general histological preparation. For example, a Bouin's fixative has been widely preferred for the reason that tissue blocks can be sufficiently fixed without deformation or contraction of tissues. Although an exact mechanism of the role of PA is yet unknown, it may be speculated that this reagent may be occluded in protein molecules and prevent a cause of tissue shrinkage, namely a formation of methylene bridge catalysed by a formaldehyde fixative. Thus PA minimizes the change of molecular conformation. This protective role in conformational alterations may also contribute to maintain an antigenicity, and so in recent studies PA has been added in fixatives for immunohistochemistry (8).

In our preliminary study, the mixture containing FA and GA gave poor results in amine fluorescence. Without PA, therefore, both reagents seemed to counteract on the formation of fluorophores derived from monoamines. In contrast, the FAGLUPAGAS fixative which was a mixture of the FAGLUPAS solution and the 
GA solution showed the best results possessing both merits of these solutions. Since the FAGLUPAS revealed a sufficient quality of amine fluorescence which was further enhanced by GA (Table 1), it was likely that, in the presence of PA, the cyclization of the fluorophores was firstly formed with the FAGLU and subsequently additional reactions occured with the GA. Similar to the case in protein, PA may preserve and insolubilize the fluorescent compound by binding it with tissue components. As an application of fixatives being omitted either one of constituents of the FAGLUPAGAS solution gave only poor results; every constituent appeared to make a multiplication effect. Although the action of PA was so powerful, a trace amount of PA remaining in sections, which could be recognized as a light staining with yellow color, resulted in a strong non-specific fluorescence. Nevertheless PA bound to protein reversibly, and that problem could be easily solved by a thorough washing. The washing had to be stopped, however, when the yellow color was sufficiently faded, otherwise prolonged treatment led to a deterioration in the intensity of the fluorescence.

In our recent study, this method has been applied in conjunction with enzyme histochemistry for acetylcholinesterase (AChE) (6). There it was possible to examine both aminergic neurons and AChE-containing neurons in the same section. Another application of this method is also available for combination with the retrograde axonal transport technique using fluorescent dyes or with immunohistochemistry.

\section{REFERENGES}

1. Ajelis, V., Björklund, A., Falck, B., Lindvall, O., Loren, I. and Walles, B.: Application of the aluminum-formaldehyde (ALFA) histofluorescence method for demonstration of peripheral stores of catecholamines and indolamines in freeze-dried paraffin-embedded tissue, cryostat sections and whole-mounts. Histochemistry 65; 1-15, 1979.

2. Corrodi, H., Hillarp, N.-A. and Jonsson, G.: Fluorescence methods for the histochemical demonstration of monoamines. Sodium borohydride reduction of the fluorescent compounds as a specificity test. J. Histochem. Cytochem. 12; 582-586, 1964.

3. Falck, B., Hillarp, N.-A., Thieme, G. and Torp, A.: Fluorescence of catecholamines and related compounds with formaldehyde. J. Histochem. Cytochem. 10; 348-354, 1962.

4. Furness, J. B., Costa, M. and Wilson, A. J.: Water-stable fluorophores, produced by reaction with aldehyde solutions, for the histochemical localization of catechol- and indolethylamines. Histochemistry 52; 159-170, 1977.

5. Fuxe, L., Hökfelt, T., Jonsson, G. and Ungerstedt, U.: Fluorescence microscopy in neuroanatomy. In Contemporary Research Methods in Neuroanatomy, ed. by W. J. H. Nauta and S. O. E. Ebbeson, Springer Verlag, Berlin, 1970, p. 275-314.

6. Kimura, H. and Maeda, T.: Aminergic and cholinergic systems in the dorsolateral pontine tegmentum. Brain Res. Bull. 8; 1982 . (in press)

7. Lindvall, O. and Björklund, A.: The glyoxylic acid fluorescence histochemical method; a detailed account of the methodology for the visualization of central catecholamine neurons. Histochemistry 39; 97-127, 1974.

8. Takeuchi, Y., Kimura, H. and Sano, Y.: Immunohistochemical demonstration of the distribution of serotonin neurons in the brainstem of the rat and cat. Cell Tissue Res. (in press) 